\title{
Direct geo-referencing of a static terrestrial laser scanner
}

\author{
Jens-André Paffenholz, Hamza Alkhatib and Hansjörg Kutterer
}

\begin{abstract}
This paper describes an adaptive extended Kalman filter (AEKF) approach for georeferencing tasks for a multi-sensor system (MSS). The MSS is a sensor fusion of a phase-measuring terrestrial laser scanner (TLS) with navigation sensors such as Global Navigation Satellite System (GNSS) equipment and inclinometers. The position and orientation of the MSS are the main parameters which are constant on a station and will be derived by a Kalman filtering process. Hence, the orientation of a TLS/MSS can be done without any demand for other artificial targets in the scanning area. However, using inclinometer measurements the spatial rotation angles about the $\mathrm{X}$ - and $\mathrm{Y}$-axis of the fixed MSS station can be estimated by the AEKF. This makes it possible to determine all six degrees of freedom of the transformation from a sensor-defined to a global coordinate system. The paper gives a detailed discussion of the strategy used for the direct geo-referencing. The AEKF for the transformation parameters estimation is presented with focus on the modelling of the MSS motion. The usefulness of the suggested approach will be demonstrated using practical investigations.
\end{abstract}

Keywords. Terrestrial laser scanning, light detection and ranging, global navigation satellite system, geo-referencing, extended Kalman filter.

\section{Introduction}

Nowadays the main characteristic of the terrestrial laser scanning technique for engineering geodesy is the immediate data acquisition in $3 \mathrm{D}$ space. This is realised with a high spatial resolution as well as with a very high frequency in a relative or local sensordefined coordinate system, respectively. The terrestrial laser scanning technique can be used in static and kinematic mode. Static scanning is characterised by one single fixed translation and orientation of the terrestrial laser scanner (TLS) in relation to an absolute or global coordinate system. For kinematic scanning, where the data acquisition is commonly reduced to $2 \mathrm{D}$ profiles, the translations and orientations are time-dependent. Hence, the transformation parameters for each profile are different in relation to each other as well as to an absolute or global coordinate system. Typically the transformation parameters are observed by navigation sensors and are estimated by a Kalman filtering process (Vennegeerts et al. 2008).

Whenever a combination of several scans from different stations into one coordinate system (registration) is required, the transformation parameters for each scan have to be determined. These parameters can be estimated by matching algorithms, e.g., the Iterative Closest Point (ICP) algorithm (Besl and McKay 1992), or by identifying several artificial control points in each scan. For an additional link to an absolute or global coordinate system (georeferencing), control points in a known geodetic datum are necessary. If the (global) coordinates of the control points also has to be determined, the procedure is more complex and time-consuming due to the additional and independent surveying task.

It is possible, however, to transfer the properties from the kinematic scanning domain - the observation of transformation parameters and the Kalman filtering process - to the static scanning domain for the registration and geo-referencing tasks of static 3D laser scans. First, there is the direct observation of the position and orientation, which can be derived by the circular motion of the fixed laser scanner about its vertical axis. For this purpose a multisensor system (MSS) is created by integrating a laser scanner and navigation sensors, which is comparable to the setup of a MSS for kinematic scanning applications as in the case of so-called mobile mapping systems (Vennegeerts et al. 2008). Second, a Kalman filtering process is used for the estimation of the transformation parameters, which are constant for the whole 3D laser scan. These parameters may also be used as appropriate start values with variance information for the matching algorithms for the registration tasks without the need of any control points. This short introduction indicates that the direct geo-referencing of static 3D laser scans offers a number of enormous advantages over the traditional way of using control points.

The paper is organised as follows. Section 2 describes the strategy for the direct geo-referencing of a TLS-based MSS. Section 3 briefly introduces the 
theory of the adaptive extended Kalman filter (AEKF). Then the proposed algorithm for the estimation of the transformation parameters is described and an outline of the modelling of the motion of the MSS is given. Practical investigations of the proposed algorithm are presented in Section 4. Finally, Section 5 summarises the results and discusses future work.

\section{Strategy}

The idea of a TLS-based MSS for direct georeferencing purposes was briefly outlined in Section 1. The main aim of the direct geo-referencing strategy is the direct observation of the required transformation parameters from the local sensor-defined coordinate system, given by the MSS, to a global coordinate system. This 3D transformation is defined by a translation vector, which is equal to the position of the MSS, and a rotation matrix, which contains the orientation of the three axes of the MSS, comparable to the roll, pitch and yaw angles in aeronautical applications. In summary this transformation has 6 degrees of freedom (DoF) which have to be observed or estimated. However, only 4 of the 6 DoF, namely the position vector as well as the azimuthal orientation (yaw), are essential. Because a fixed terrestrial MSS can be oriented to the direction of gravity, the spatial rotations (roll and pitch) about the $\mathrm{X}$ - and $\mathrm{Y}$-axis of the MSS can be minimised.

In the following section, the technique for the direct geo-referencing of a TLS-based MSS, which is realised as an adapted sensor-driven method at the Geodetic Institute of the Gottfried Wilhelm Leibniz Universität Hannover (GIH), will be described. The technique can be divided into a sensor fusion part and the algorithm for the estimation of the transformation parameters, which will be described in Section 3.

\subsection{Senor fusion in the MSS}

As already mentioned in Section 1, the MSS is established by a fusion of a phase-measuring TLS, which is the main sensor, and additional navigation sensors to observe the transformation parameters. The operation of the laser scanner should neither be restricted nor disturbed by the additional sensors. Hence, one should take advantage of the individual characteristics of any sensor in the MSS. Here one can point out the use of the constant rotation of the laser scanner about its vertical axis as a time and orientation reference. Due to the TLS having a high frequency data acquisition rate, which is usually more than $10 \mathrm{~Hz}$ for the phase-measuring TLS (e.g. Zoller+Fröhlich Imager 5006), and due to the need for reliable transformation parameters, the availability of data rates of at least $10 \mathrm{~Hz}$ for the additional sensors is required. Indispensable in the MSS is the synchronisation of all sensors so that the individual measurements can be related to each other in a temporal sense. Therefore it is useful to introduce a unique time reference in the MSS. The most suitable way to do this is to use the Global Positioning System (GPS) time as reference because it is instantaneously available from the Global Navigation Satellite System (GNSS) observations.

As a minimum one GNSS equipment is required as additional sensor. The mounting of two GNSS antennae on top of the laser scanner leads to different approaches within the scope of the GNSS analysis. One approach is the calculation of a single baseline for each antenna. The second approach is the evaluation of the relative baseline between the two antennae. The last is known in literature as GNSS compass; e.g. see Teunissen et al. (2010). Nevertheless, if one or two GNSS antennae are used, the trajectory of an antenna reference point (ARP) is a space curve which is described by the circular motion of the laser scanner about its vertical axis. For the first realisation of a MSS for direct geo-referencing at the GIH only one GNSS equipment was used. This leads to only 4 of the 6 DoF being determinable. Additionally, a horizontally mounted MSS is assumed and any residual divergence of the orientation to the direction of gravity will be neglected. For further details, especially about the synchronisation of the MSS, please refer to Paffenholz and Kutterer (2008).

In order to optimise the direct geo-referencing strategy, some sensor modifications in the MSS were carried out. In a first step the MSS is extended with additional navigation sensors - in this case inclinometers - to estimate the remaining $2 \mathrm{DoF}$ (the spatial rotations about the $\mathrm{X}$ - and $\mathrm{Y}$-axis of the MSS). Two single-axis inclinometers were mounted next to the GNSS antenna on top of the laser scanner, each of them observing one spatial rotation about the X-and Y-axis (see Figure 1). For synchronisation purposes a real-time computer with integrated analog-digital (A/D) converter is used. The utilisation of integrated inclinometers in a new laser scanner series instead of additional sensors would also be possible. 


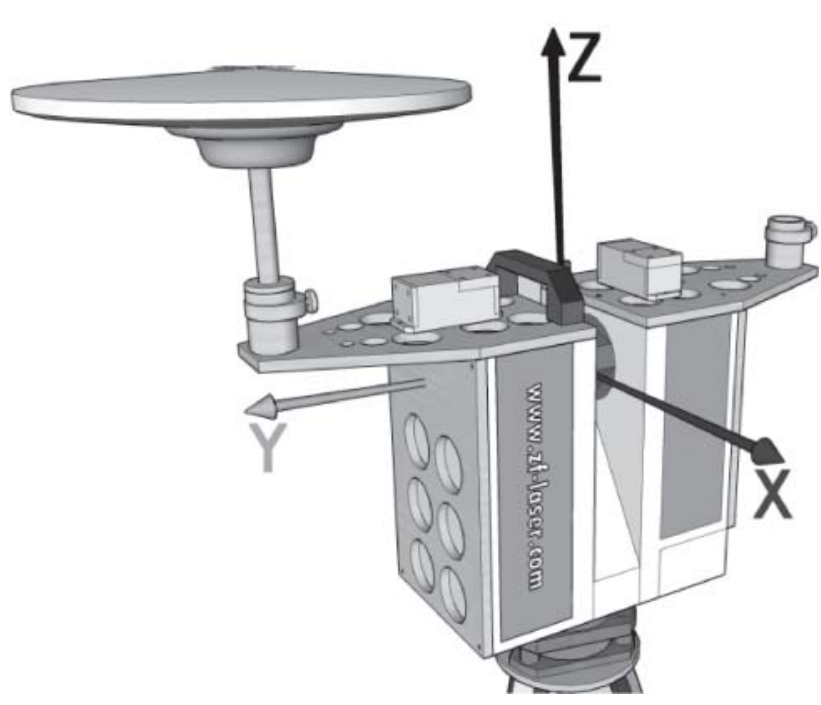

Figure 1: Setup of the MSS equipped with one GNSS antenna and two external inclinometers on top of a phasemeasuring TLS.

A further modification of the MSS compared to Paffenholz et al. (2009) was to use the horizontal motor steps of the laser scanner as additional input data. Thereby measured information about the rotation of the laser scanner is available. The synchronisation of this new data source is directly available from the general synchronisation pulse of the laser scanner which corresponds to the progress in the rotation of the laser scanner about its vertical axis.

The described sensor modification and the availability of additional data led to significant modifications of the algorithm for the estimation of the transformation parameters. In addition, and due to the consideration of all DoF of the transformation from the local to the global coordinate system, a more accurate estimation of the unknown parameters is expected.

\subsection{Overview of the strategy}

In Figure 2 a schematic overview of the strategy from the data acquisition to the estimation of the transformation parameters is given. In order to simplify the complex strategy the whole procedure is structured into five parts.

Part I is the data acquisition module of the MSS. The four sensor types are the GNSS equipment, the terrestrial laser scanner with integrated inclinometer, the external inclinometer, and the real-time computer with $\mathrm{A} / \mathrm{D}$ converter. For each sensor type in- dividual pre-processing is performed. For the GNSS equipment the analysis is performed using commercial software, e.g. Wal by Wanninger (2009) or Geonap by $\mathrm{Geo}++{ }^{\circledR}$ (Wübbena 1989). The line synchronisation pulses of the laser scanner, registered with the event marker input, are analysed with respect to data gaps. The time stamp of the data is already the GPS time reference. For the TLS the raw values of the horizontal motor steps are extracted from the stored 3D point cloud. They correspond to the number of $2 \mathrm{D}$ profiles within the 3D point cloud as well as to the number of line synchronisation pulses. The processing of the registered inclinations from the integrated sensor is a topic for future work. The analogue data streams of the external inclinometers are processed by the A/D converter within the real-time computer. All data streams that come into the realtime computer are marked with an internal time stamp of the computer. Besides the inclinations, the pulse per second (PPS) signal and a National Marine Electronics Association (NMEA) string, containing the UTC time information of the GNSS receiver, as well as the line synchronisation pulses, are input to the real-time computer. The data delivered by the GNSS receiver is pre-processed in a way that for each PPS the corresponding GPS time stamp is generated by the UTC information extracted from the NMEA string. The line synchronisation pulses are registered twice, once by the GNSS receiver (event marker input) and once by the realtime computer. The data stored by the GNSS receiver is used for subsequent data processing. The registered pulses by the real-time computer are used as backup.

Part II is concerned with the data synchronisation of the MSS. The GPS time is the unique time scale of the MSS. Therefore, the data which is not related to the time reference by a GPS time stamp has to be synchronised. This fact is true for all data streams of the real-time computer, which are marked with an internal time stamp. The relationship between the internal time stamp and the GPS time is established by the data stream of the GNSS receiver. Hence, the introduction of the required GPS time stamp for the inclinations, as well as for the registered line synchronisation pulses (\# of scan profiles), is possible.

Up to Part III all data sources are marked with the GPS time stamp. In this data fusion step for each 2D profile of the 3D point cloud the corresponding 3D position of the ARP and inclination has to be determined. Therefore, the data is interpolated with respect to the time stamp of each $2 \mathrm{D}$ profile. 


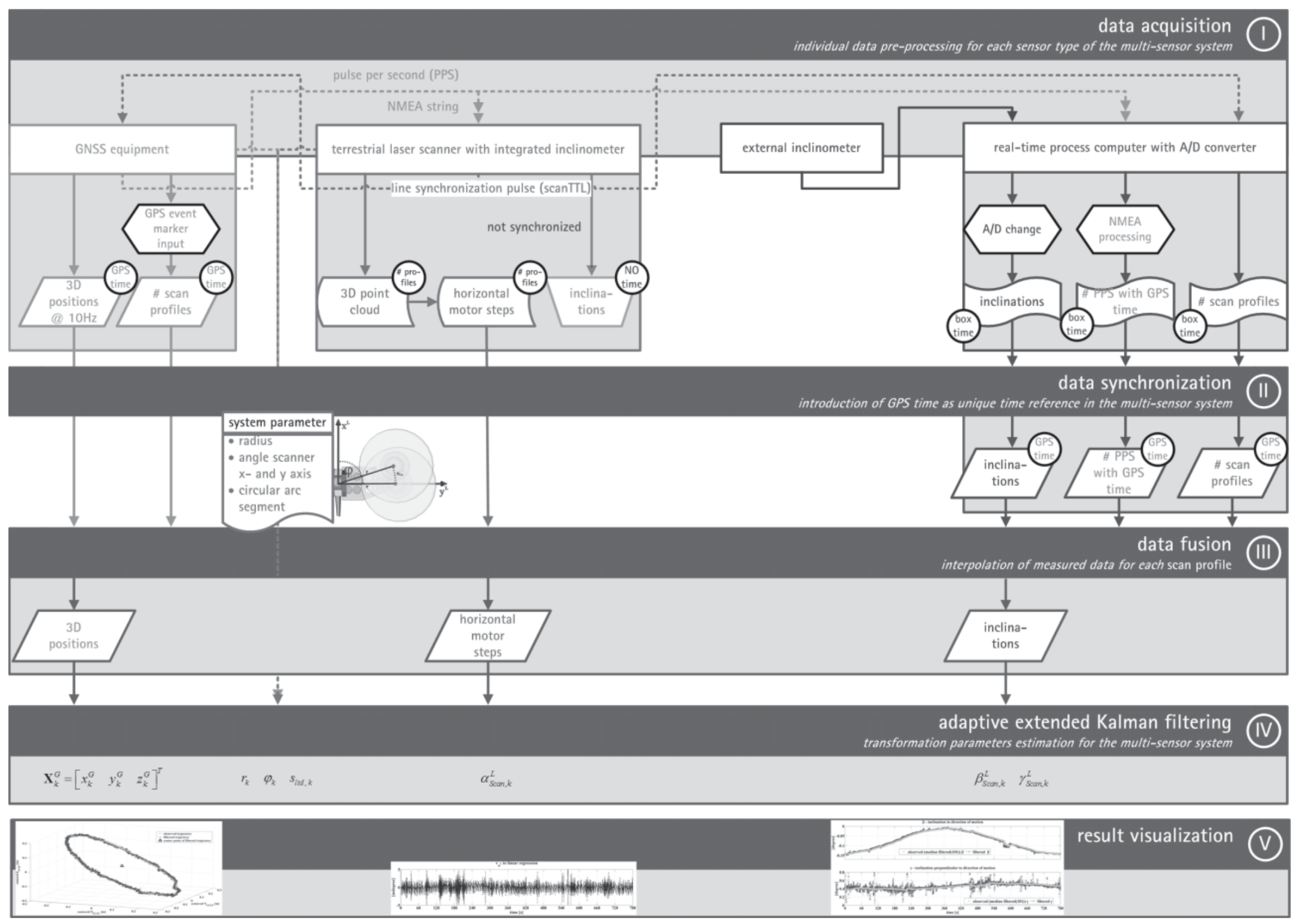

Figure 2: Schematic overview of the strategy from data acquisition to the estimation of the transformation parameters. 
Part IV is the core data analysis module. Using an AEKF the estimation of the transformation parameters is performed. Besides the already mentioned observations, three system parameters are integrated into the AEKF as adaptive parameters. These time invariant parameters are determined in an independent procedure with high accuracy using a laser tracker. More details about the modelling of the motion of the MSS, the elements of the system equations and the measurement model are given in Section 3. The achieved results are discussed in Section 4.

Part V indicates further investigations, like comparisons of selected transformed artificial control points with global reference coordinates. In future work the transformation of the whole 3D point cloud, from the local sensor-defined to a global coordinate system, will be performed.

\section{Proposed algorithm and modelling of the motion of the MSS}

In Section 2 the strategy for the direct georeferencing of a TLS-based MSS was described. Section 3.1 gives a brief survey of the theory of the extended Kalman Filter (EKF). The algorithm for the estimation of the transformation parameters will be described in Section 3.2. The modelling of the motion of the MSS will be outlined in Section 3.3.

\subsection{Theory of the first order EKF with adaptive parameters}

The modelling of trajectories of moving vehicles often leads to non-linearities in the system equations of the Kalman filter (KF). For example, Aussems (1999) describes the trajectory of a vehicle with a refined model of consecutive arcs. The circular motion of the TLS about its vertical axis within the static MSS can be described in a comparable model; refer to Section 3.3. In both cases, the functional relationship between the vehicle as well as the MSS coordinates and the other state parameters is non-linear (Simon 2006). However, the state estimation within a KF is optimal only in the case of linear state space systems. The EKF can account for non-linearities in the system and measurement equations. Further details about the EKF can be found in Simon (2006), for example. The incorporation of additional parameters in the dynamic model may improve the filtering and brings the model closer to reality (Eich- horn 2007). The EKF with adaptive parameters (AEKF) is well known in the literature as "dual estimation", see Nelson (2000), for example.

Defining the problem of a first order EKF with adaptive parameters for the non-linear case the general state vector $\mathbf{x}_{g, k} \in \mathbb{R}^{n_{x g}}$, with $n_{x_{g}}$ the number of parameters, and the adaptive state vector $\mathbf{x}_{a, k} \in \mathbb{R}^{n_{x_{a}}}$, with $n_{x_{a}}$ the number of adaptive parameters, as well as the time index $k$ are given.

The state vector $\mathbf{X}_{k}$, composed of $\mathbf{x}_{g, k}$ and $\mathbf{x}_{a, k}$, and its variance-covariance matrix $(\mathrm{vcm}) \Sigma_{\mathbf{X}_{k} \mathbf{x}_{k}}$ for a non-linear problem are given by:

$$
\begin{aligned}
\mathbf{X}_{k} & =\left[\begin{array}{ll}
\mathbf{x}_{g, k} & \mathbf{x}_{a, k}
\end{array}\right]^{T} \text { and } \\
\Sigma_{\mathbf{X}_{k} \mathbf{X}_{k}} & =\left[\begin{array}{cc}
\Sigma_{\mathbf{x}_{g, k} \mathbf{x}_{g, k}} & \mathbf{0} \\
\mathbf{0} & \Sigma_{\mathbf{x}_{a, k} \mathbf{x}_{a, k}}
\end{array}\right] .
\end{aligned}
$$

The known non-linear function $\mathbf{f}($.$) is composed of:$

$$
\mathbf{f}=\left[\begin{array}{ll}
\mathbf{f}_{g}\left(\mathbf{x}_{g, k}, \mathbf{u}_{k}, \mathbf{w}_{k}\right) & \mathbf{f}_{a}\left(\mathbf{x}_{a, k}, \mathbf{u}_{k}, \mathbf{w}_{k}\right)
\end{array}\right]^{T} .
$$

Here $\mathbf{f}_{g}($.$) , so called dynamic model, is a known$ non-linear function of the general parameters and $\mathbf{f}_{a}($.$) is a known non-linear function of the adaptive$ parameters, $\mathbf{u}_{k}$ is a vector of known (deterministic) input and $\mathbf{w}_{k}$ is the vector of the process noise, which is caused by imperfect modelling effects and other disturbances in the motion model which can be assumed to normally distributed $\mathbf{w}_{k} \sim \mathscr{N}\left(\mathbf{0}, \Sigma_{\mathbf{w}_{k}, \mathbf{w}_{k}}\right)$. The main aim of the filtering is to get optimal $\mathbf{x}_{g, k+1}$ and $\mathbf{x}_{a, k+1}$ from the observations $\mathbf{y}_{k+1} \in \mathbb{R}^{n_{y}}$, $\mathbf{x}_{g, k} \in \mathbb{R}^{n_{x_{g}}}$ and $\mathbf{x}_{a, k} \in \mathbb{R}^{n_{x_{a}}}$ recursively, where $n_{y}$ is the dimension of the measurement model:

$$
\mathbf{y}_{k+1}=\mathbf{h}\left(\mathbf{x}_{g, k+1}, \mathbf{x}_{a, k+1}, \mathbf{v}_{k+1}\right) .
$$

Here $\mathbf{h}($.$) is a (usually) known non-linear function,$ and $\mathbf{v}_{k+1}$ is the measurement noise vector, which is assumed to be independent and uncorrelated with a known probability density function, typically the Gaussian distribution.

The Algorithm 1 gives a brief overview of the dual estimation with first order EKF. The transition and design matrices $\mathbf{F}_{k}$ and $\mathbf{H}_{k+1}$ in the Algorithm 1 are defined as:

$$
\mathbf{F}_{k}=\left.\frac{\partial \mathbf{f}}{\partial \mathbf{x}}\right|_{\mathbf{x}=\mathbf{x}_{k}(+)} \quad \text { and } \quad \mathbf{H}_{k+1}=\left.\frac{\partial \mathbf{h}}{\partial \mathbf{x}}\right|_{\mathbf{x}=\mathbf{x}_{k+1}(-)},
$$

respectively. The Taylor series are evaluated for the transition matrix $\mathbf{F}_{k}$ at the previous epoch $\mathbf{x}_{k}(+)$ and at the current epoch $\mathbf{x}_{k+1}(-)$ for the design matrix $\mathbf{H}_{k+1}$. Please note that the functions $\mathbf{f}($.$) and \mathbf{h}($. are both time invariant. 


\subsection{Algorithm for the estimation of the transformation parameters}

The estimation of the transformation parameters according to the first realisation of the MSS, which was described in Section 2.1, is carried out in a two step procedure. First the 3D positions are projected onto a best-fitting plane, and then a best-fitting circle through the projected positions is estimated. These two parameter estimations are computed by means of a least-squares adjustment. Furthermore, in both steps an outlier detection algorithm (Baarda's data snooping) is applied. In order to derive an azimuthal orientation for each scan line of the 3D laser scan as well as to estimate the position of the MSS, the space curve parameters (centre point and radius), and the adjusted observations, respectively, are required. For further details please refer to Paffenholz and Kutterer (2008). The main disadvantage of this two-step procedure is that the setup of two separated adjustments is required. In addition, the data acquisition must be completed before performing the processing steps.

According to the sensor modifications in the MSS and the additionally available data sources, the estimation of all $6 \mathrm{DoF}$ of the transformation is possible. This leads to a new analysis strategy with vital modifications of the algorithm for the estimation of the transformation parameters. The new algorithm was developed in a closed form on the basis of a EKF. This EKF-based algorithm resolves one of the above mentioned drawbacks. On the one hand the KF allows for real-time processing. On the other hand the parameter estimation will be less sensitive to outliers. The main aim of a KF is the optimal combination of given physical information about a system and external observations of its state. To deal with non-linearities in the system and measurement equations an EKF is used to estimate the transformation parameters of the MSS. Another promising approach for a non-linear state estimation is the combination of Sequential Monte Carlo filtering (also known as particle filter) and an EKF, which was proposed by Alkhatib et al. (2010). The main benefit of the proposed approach is the better performance in case of high-nonlinear space state equations. As already mentioned, an improvement of the dynamic model of the EKF can be achieved by augmenting the EKF with adaptive parameters. These parameters are time invariant and systemspecific with well-known initial values. In comparison with the algorithm described in Paffenholz et al. (2009), the filter setup was modified and optimised
Algorithm 1: First order EKF with adaptive parameters

\section{Initialisation}

I Initial system state $\mathbf{X}_{k=0}$ and its vem $\Sigma_{\mathbf{X}_{k=0} \mathbf{X}_{k=0}}$

for $k=1,2, \ldots, n_{\text {epochs }}$ do

Prediction step

Input: $\hat{\mathbf{x}}_{g, k}, \hat{\mathbf{x}}_{a, k}, \mathbf{u}_{k}$

Output: $\hat{\mathbf{X}}_{k+1}(-), \Sigma_{\hat{\mathbf{X}}_{k+1} \hat{\mathbf{X}}_{k+1}(-)}$

$$
\hat{\mathbf{X}}_{k+1}(-)=\mathbf{f}\left(\hat{\mathbf{x}}_{g, k}, \hat{\mathbf{x}}_{a, k}, \mathbf{u}_{k}, k\right)
$$$$
=\left[\begin{array}{c}
\mathbf{f}_{g}\left(\hat{\mathbf{x}}_{g, k}, \mathbf{u}_{k}, k\right) \\
\mathbf{f}_{a}\left(\hat{\mathbf{x}}_{a, k}, \mathbf{u}_{k}, k\right)
\end{array}\right]
$$

$\Sigma_{\hat{\mathbf{x}}_{k+1} \hat{\mathbf{x}}_{k+1}(-)}=\mathbf{F}\left(\hat{\mathbf{x}}_{g, k}, \hat{\mathbf{x}}_{a, k}, \mathbf{u}_{k}, k\right) \cdot \Sigma_{\hat{\mathbf{X}}_{k+1} \hat{\mathbf{X}}_{k+1}}$ $\cdot \mathbf{F}^{T}\left(\hat{\mathbf{x}}_{g, k}, \hat{\mathbf{x}}_{a, k}, \mathbf{u}_{k}, k\right)+\Sigma_{\mathbf{w}_{k}, \mathbf{w}_{k}}$

Update step

$$
\begin{aligned}
& \text { Input: } \hat{\mathbf{X}}_{k+1}(-), \mathbf{y}_{k+1} \\
& \text { Output: } \hat{\mathbf{X}}_{k+1}(+), \Sigma_{\hat{\mathbf{X}}_{k+1} \hat{\mathbf{X}}_{k+1}}(+) \\
& \mathbf{K}_{k+1}=\Sigma_{\hat{\mathbf{X}}_{k+1} \hat{\mathbf{X}}_{k+1}(-)} \cdot \mathbf{H}_{k+1}^{T} \\
& \cdot\left(\Sigma_{\mathbf{y}_{k+1}, \mathbf{y}_{k+1}}+\mathbf{H}_{k+1}\right. \\
& \left.\cdot \Sigma_{\hat{\mathbf{X}}_{k+1} \hat{\mathbf{X}}_{k+1}(-)} \cdot \mathbf{H}_{k+1}^{T}\right)^{-1} \\
& \hat{\mathbf{X}}_{k+1}(+)=\hat{\mathbf{X}}_{k+1}(-)+\mathbf{K}_{k+1} \\
& \cdot\left(\mathbf{y}_{k+1}-\mathbf{h}\left(\hat{\mathbf{X}}_{k+1}(-)\right)\right) \\
& \Sigma_{\hat{\mathbf{X}}_{k+1} \hat{\mathbf{X}}_{k+1}}(+)=\Sigma_{\hat{\mathbf{X}}_{k+1} \hat{\mathbf{X}}_{k+1}(-)}-\mathbf{K}_{k+1} \\
& \cdot\left(\Sigma_{\mathbf{y}_{k+1}, \mathbf{y}_{k+1}}+\mathbf{H}_{k+1}\right. \\
& \left.\Sigma_{\hat{\mathbf{X}}_{k+1} \hat{\mathbf{x}}_{k+1}(-)} \cdot \mathbf{H}_{k+1}^{T}\right) \cdot \mathbf{K}_{k+1}^{T}
\end{aligned}
$$

end

following the first experiences with the new analysis strategy. This will be discussed in detail in Section 4 .

\subsection{Modelling of the motion of the MSS}

The trajectory of the MSS can be parametrised by a circle in $3 \mathrm{D}$ space. In comparison to the general trajectory state estimation in engineering geodesy, e.g. Aussems (1999), this parameterisation is possible due to the circular motion of the ARP. This circular motion is caused by the constant rotation of the laser scanner about its vertical axis and the fixed positioning of the GNSS antenna on top of the rotating part of the laser scanner. 


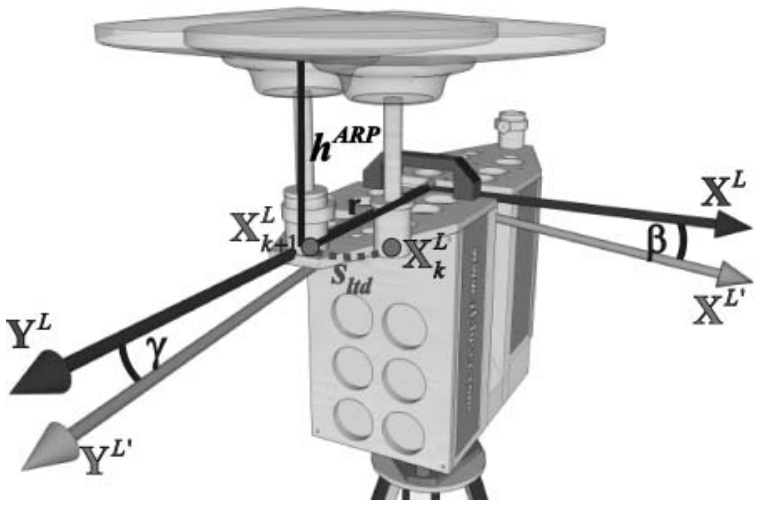

Figure 3: Side view of the planar motion of the ARP and residual divergences $\beta$ and $\gamma$ of the orientation to the direction of gravity.

The modelling of the motion of the MSS starts with a local sensor-fixed coordinate system with the upper index $L$ (see Figure 3, in black), which is defined by the $\mathrm{X}$ - and $\mathrm{Y}$-axis of the laser scanner. In general there will be a residual divergence of the orientation to the direction of gravity, which leads to the coordinate system with the upper index $L^{\prime}$ (see Figure 3, in gray). In order to describe the planar motion of the ARP with respect to the coordinate system $L$, the residual spatial rotations about the $\mathrm{X}$-axis $\beta$ - in the scan direction - and about the $\mathrm{Y}$-axis $\gamma$ - perpendicular to the scan direction - have to be considered. These two residual spatial rotations or inclinations are introduced as additional state parameters in the AEKF, to augment the global position of the ARP $\mathbf{X}^{G}=\left[\begin{array}{lll}x^{G} & y^{G} & z^{G}\end{array}\right]^{T}$ and the local orientation $\alpha_{\text {Scan }}^{L}$. Figure 4 shows the planar motion of the ARP, described by the system parameters radius $r$ and circular arc segment $s_{l t d}$. In addition to the radius and circular arc segment, the angle between $\mathrm{X}$ - and $\mathrm{Y}$-axis $\varphi$ is introduced as an adaptive parameter in the AEKF. These three system parameters are determined in a system calibration procedure for the MSS by laser tracker measurements.

The local planar motion of the ARP, modelled by the state parameters $\beta$ and $\gamma$ and the adaptive parameters $r$ and $s_{l t d}$ for an epoch $k$, is described as:

$$
\begin{aligned}
\mathbf{X}_{k+1}^{L}= & {\left[\begin{array}{lll}
x_{k+1}^{L} & y_{k+1}^{L} & z_{k+1}^{L}
\end{array}\right]^{T} } \\
= & {\left[\begin{array}{c}
r_{k} \cdot \cos \left(\gamma_{\text {Scan }, k}^{L}\right) \cdot \sin \left(\frac{s_{l d, k}}{r_{k}}\right) \\
r_{k} \cdot \cos \left(\gamma_{\text {Scan }, k}^{L}\right) \cdot \cos \left(\frac{\left(s_{\text {ld }, k}\right.}{r_{k}}\right) \\
s_{\text {ltd }, k} \cdot \sin \left(\beta_{\text {Scan }, k}^{L}\right)
\end{array}\right] . }
\end{aligned}
$$

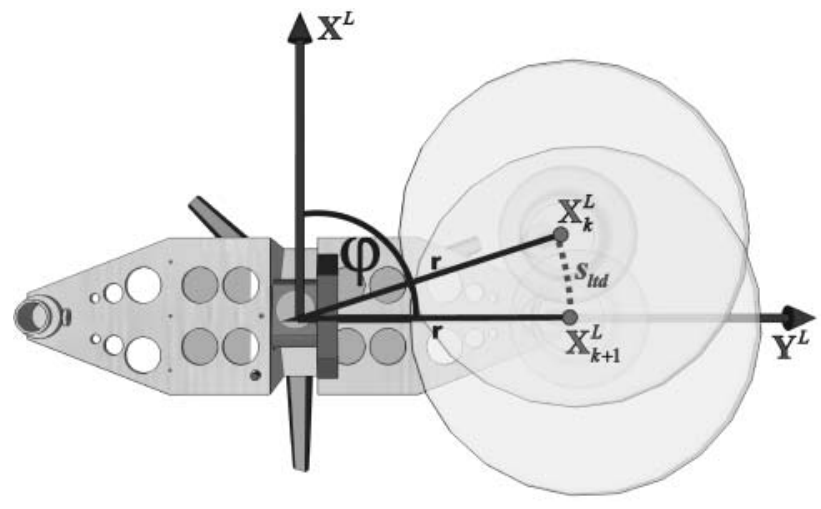

Figure 4: Top view of the planar motion of the ARP and system parameters $r, \varphi$ and $s_{l t d}$ (adaptive parameters in the AEKF).

The orientation change of the ARP between two epochs is expressed by the circular arc segment divided by the radius. This is in contrast to the former approach described in Paffenholz et al. (2009), where the orientation change was expressed by the velocity $v$ and the time interval $\Delta T$ divided by the radius $r$. This parameterisation is not suitable for the non-accelerated motion of the MSS because the velocity parameter $v$ cannot be measured reliably. To avoid the use of pseudo-observations for the velocity parameter, as in the former approach, the orientation change is expressed by the circular arc segment as mentioned above.

For the description of the local planar motion of the ARP from one epoch to the next, the scanner origin has to be taken into account. Therefore the translation between the actual ARP position and the scanner origin is expressed by:

$$
\mathbf{X}_{S c a n N, k}^{\text {GNSS }}=\left[\begin{array}{c}
-r_{k} \cdot \cos \left(\varphi_{k}\right) \\
r_{k} \cdot \sin \left(\varphi_{k}\right) \\
h^{A R P}
\end{array}\right] .
$$

The translations in $\mathrm{X}$ - and $\mathrm{Y}$-axis are given by the adaptive parameters radius and the angle between the $\mathrm{X}$ - (scan direction) and $\mathrm{Y}$-axis (in direction to the mounted GNSS antenna), see Figure 4. The value for the angle $\varphi$ depends on the antenna position on the scanner - positive $\mathrm{Y}$-axis as shown in Figure 3 and 4 or negative $\mathrm{Y}$-axis - and is nearly 100 gon or 300 gon, respectively. The translation in $z$-axis $h^{A R P}$ is given by the antenna height which can already be considered in the GNSS analysis and has then to be set to zero.

To complete the description of the local planar motion of the ARP, the local orientation $\alpha_{\text {Scan }}^{L}$, which is 
updated by $\alpha_{\text {Scan }, k+1}^{L}=\alpha_{\text {Scan }, k}^{L}+\frac{s_{l d d, k}}{r_{k}}$, has to be considered:

$$
\Delta \mathbf{X}_{k+1}^{L}=\mathbf{R}_{\text {Scan } N}^{L}\left(\alpha_{\text {Scan }, k+1}^{L}\right) \cdot\left[\mathbf{X}_{k+1}^{L}-\mathbf{X}_{\text {Scan }, k}^{G N S S}\right] .
$$

The transformation of the local planar motion given in equation (8) is the last step in modelling the motion of the ARP in the local coordinate system, besides the consideration of the ARP position at epoch $k$ :

$$
\mathbf{X}_{k+1}^{G}=\mathbf{X}_{k}^{G}+\mathbf{R}_{\alpha^{G}}^{G}(\lambda, \phi) \cdot \mathbf{R}_{L}^{\alpha^{G}}\left(\alpha^{G}\right) \cdot \Delta \mathbf{X}_{k+1}^{L},
$$

where $\mathbf{R}_{L}^{\alpha^{G}}\left(\alpha^{G}\right)$ defines the global azimuthal orientation and $\mathbf{R}_{\alpha^{G}}^{G}(\lambda, \phi)$ describes the transformation from the local to the global coordinate system. The value for the initial global azimuthal orientation $\alpha^{G}$, the longitude $\lambda$, and latitude $\phi$ of the MSS are calculated as mean values of all trajectory points. The state vector $\mathbf{X}$ for an epoch $k$ is finally given as:

$$
\begin{aligned}
& \mathbf{X}_{k}=\left[\begin{array}{lll}
\mathbf{x}_{g, k} & \mid \mathbf{x}_{a, k}
\end{array}\right]^{T} \\
& =\left[\begin{array}{llll}
\mathbf{X}_{k}^{G} & \alpha_{\text {Scan }, k}^{L} & \beta_{\text {Scan }, k}^{L} & \gamma_{\text {Scan }, k}^{L}
\end{array} \mid\right. \\
& \left.\begin{array}{lll}
r_{k} & \varphi_{k} & s_{l t d, k}
\end{array}\right]^{T}
\end{aligned}
$$

where $\mathbf{x}_{g, k}$ is the general state vector and $\mathbf{x}_{a, k}$ the adaptive state vector.

The measurement model is defined by the observation vector of the GNSS positions $\overline{\mathbf{X}}^{G}=$ $\left[\begin{array}{lll}\bar{x}^{G} & \bar{y}^{G} & \bar{z}^{G}\end{array}\right]^{T}$, the value of the horizontal motor step of the laser scanner $\bar{\alpha}_{S c a n}^{L}$, and the measurements of the inclination sensors $\bar{\beta}_{\text {Scan }}^{L}$ and $\bar{\gamma}_{\text {Scan }}^{L}$. It can be expressed by the following linearised measurement equations:

$$
\mathbf{y}_{k+1}=\left[\begin{array}{cc}
\underline{\mathbf{I}} & \underline{\mathbf{0}} \\
6,6
\end{array}\right] \cdot \mathbf{X}_{k}+\mathbf{v}_{k+1}
$$

where $\mathbf{y}=\left[\begin{array}{llllll}\bar{x}^{G} & \bar{y}^{G} & \bar{z}^{G} & \bar{\alpha}_{\text {Scan }}^{L} & \bar{\beta}_{\text {Scan }}^{L} & \bar{\gamma}_{\text {Scan }}^{L}\end{array}\right]^{T}$ denotes the vector of the observations and $\mathbf{v}$ are the corresponding residuals.

\section{Practical investigations of the proposed algorithm}

This section is divided into four parts. First, the general observation strategy for the practical investigations is outlined. Second, a brief overview of the GNSS analysis is given. Third, a dataset with a MSS setup similar to Figure 1 is investigated. The measurements of this dataset, called $G I H$, were performed in front of the GIH building in Hanover using only one fixed station. Fourth, a dataset with an additional 3D positioning method next to the GNSS measurements will be discussed. This dataset, called
Welfenschloss, was captured in front of the Welfenschloss, the main building of the Leibniz Universität Hannover (LUH).

\subsection{General observation strategy for the two practical investigations}

In the present system only one GNSS equipment is used to observe the rotation of the TLS around its vertical axis. For the dataset Welfenschloss the rotation was additionally tracked by a synchronised tacheometer. The simultaneously captured data of all sensors, which were already described in Section 2.2 (also including the tacheometer measurements for the dataset Welfenschloss), are the only environment-specific input data for the AEKF. The adaptive parameters are estimated in a calibration procedure under laboratory conditions with a laser tracker. The required values for the connection of the local and the global coordinate system are calculated using the kinematic GNSS observations as well as local reference points for the dataset Welfenschloss. For the longitude and latitude this is a feasible solution due to the large number of $3 \mathrm{D}$ positions. The absolute orientation is also determinable in this way, even though the reliability of the result is not optimal. One can obtain results for the metric uncertainty caused by the global azimuth in the range of a few centimetres for a distance of about $35 \mathrm{~m}$ (Paffenholz and Kutterer 2008).

\subsection{GNSS analysis strategy}

The kinematic GNSS analysis strategy depends on the number of GNSS equipments (receiver and antenna) used in the MSS. Each ARP (of the eccentrically mounted antenna on top of the TLS) is subject to a circular motion due to the rotation of the TLS (around its vertical axis). The current setup of the MSS consists of one GNSS equipment which operates with a data acquisition rate of $10 \mathrm{~Hz}$. If two GNSS equipments were used in the MSS, the GNSS analysis can be done in two different ways. One way is the calculation of one single baseline for each equipment. The combination of the two ARP trajectories has then to be realised in a subsequent filter algorithm. The other way requires the calculation of a relative baseline between the two GNSS antennae installed on top of the laser scanner. This is comparable to the GNSS compass approach, e.g. given in Teunissen et al. (2010). Within the filter algorithm there is no combination step necessary. Apart from the $3 \mathrm{D}$ positions of the ARP trajectory, 
the corresponding variance-covariance matrices are used within the filter algorithm to derive the position and, especially, the orientation, of the MSS.

For the kinematic GNSS data processing, several approaches are possible. The common characteristic of all approaches is the data post-processing. In addition, a GNSS reference station is required to obtain precise 3D positions for the circular motion of the ARP, as well as for an additional transformation to a global coordinate system. Real-time processing would be possible. But however, the potential to determine reliable transformation parameters with the real-time derived $3 \mathrm{D}$ positions has to be investigated in future. Different post-processing approaches with regard to the GNSS reference station used are possible:

a) Use of a service, e.g. the satellite positioning service of the German state survey SAPOS or the satellite positioning services ascos operated by AXIO-NET GmbH; if necessary in terms of a virtual reference station.

b) Set up of an own reference station with known coordinates close to the scanning scene. The coordinates can be determined (if needed) with a service discussed in the first approach.

The 3D positions and their variances are of great importance in deriving the quality of the position and orientation information of the MSS. On the one hand the error budget of the GNSS components in combination with the environment has to be considered. These are errors such as near-field effects caused by the antenna adaptor (made of aluminium) on the laser scanner, or possibly multipath from the surrounding area. In addition, the data acquisition principle of the MSS has to be considered with respect to the rotation of the laser scanner about its vertical axis. This could contribute another error caused by the alternating antenna orientation. A solution may be possible by the introduction of azimuth dependent phase centre variations offsets. On the other hand, the kinematic GNSS data processing itself affects the quality of the 3D positions and the derived position and orientation of the MSS.

\subsection{Example dataset GIH}

Figures 5 and 6 show a sample of the estimated state parameters from the AEKF described in Section 3. The elements of the matrix of the process noise in the AEKF are rather pessimistic values, mainly based on experiences with the different sensors and the first practical datasets. For the data acquisition,

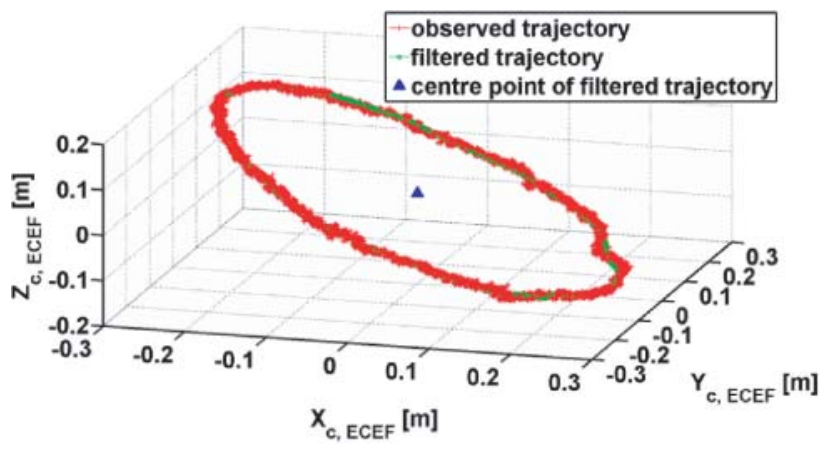

Figure 5: Observed and filtered trajectories of the ARP in an ECEF coordinate system reduced to the centre point.

a phase-measuring laser scanner Zoller+Fröhlich Imager 5006, one Trimble GPS receiver R5700 with Geodetic Zephyr antenna, and two Schaevitz LSOC$1^{\circ}$ inclinometers were used.

In Figure 5 the filter effect is clearly visible for the first three state parameters in an Earth Centred, Earth Fixed (ECEF) coordinate system. The blue triangle in the middle of the trajectory represents the centre point of the filtered positions or translation vector of the MSS, respectively. In top of Figure 6, the the residuals obtained from a linear regression of the filtered $\alpha^{L}$ are shown. Due to the constant rotation of the TLS about its vertical axis, one expects a linear relationship between $\alpha^{L}$ and time. Therefore, the residuals are quality indicators. The oscillations of the residuals as well as their magnitude is attributable to the resolution of the horizontal motor of $0.0018^{\circ}$, see Zoller + Fröhlich $\mathrm{GmbH}$ (2007). In the middle and bottom part of Figure 6, the filter effect is clearly visible for the filtered inclinations. In the middle part of Figure 6, the jump in $\beta^{L}$ in the time range $500-600 \mathrm{~s}$ is interpreted as an error due to the equipment, and not due to an incorrect filtering process. This error did not appear in any other experiment. The significantly higher noise level for $\gamma^{L}$ (bottom of Figure 6) can be explained by the used sensor. This is not a general problem of the used sensor type Schaevitz LSOC-1 $1^{\circ}$ in connection with rotating laser scanner. Rather, it is a phenomenon of one of our sensors at the GIH, which was noted in several projects in the past.

Table 1 summarises the statistical values of the differences between filtered and observed state vector elements. Note that for the ARP positions the Euclidean distance is used as the representative value to illustrate the filtering results in the $3 \mathrm{D}$ space, 

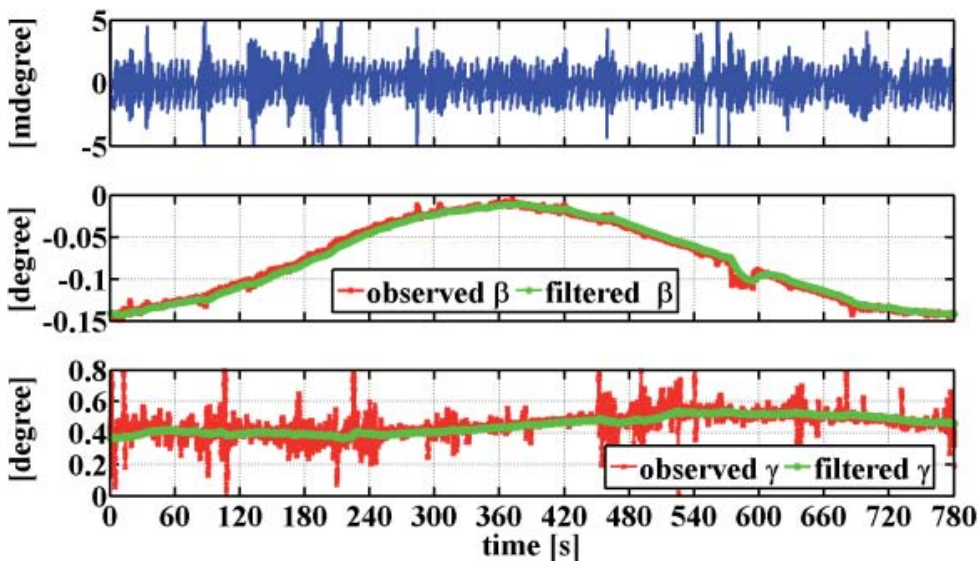

Figure 6: Top: residuals obtained from a linear regression of the filtered $\alpha^{L}$; middle: observed and filtered inclinations in direction of motion $\left(\beta^{L}\right)$; bottom: observed and filtered inclinations perpendicular to direction of motion $\left(\gamma^{L}\right)$.
Table 1: Statistical values of the difference between the filtered and observed state vector.

\begin{tabular}{lllll}
\hline & $\min$ & $\max$ & $\operatorname{mean}$ & std \\
\hline$\left\|\mathbf{X}^{G}-\overline{\mathbf{X}}^{G}\right\|$ & $0.1 \mathrm{~mm}$ & $36.5 \mathrm{~mm}$ & $5.9 \mathrm{~mm}$ & $3.4 \mathrm{~mm}$ \\
$\alpha_{\text {Scan }}^{L}-\bar{\alpha}_{\text {Scan }}^{L}$ & $-1^{\prime} 35.4^{\prime \prime}$ & $1^{\prime} 18.8^{\prime \prime}$ & $0.003^{\prime \prime}$ & $4.7^{\prime \prime}$ \\
$\beta_{\text {Scan }}^{L}-\bar{\beta}_{\text {Scan }}^{L}$ & $-54.0^{\prime \prime}$ & $1^{\prime} 50.9^{\prime \prime}$ & $-0.002^{\prime \prime}$ & $14.4^{\prime \prime}$ \\
$\gamma_{\text {Scan }}^{L}-\bar{\gamma}_{\text {Scan }}^{L}$ & $-31^{\prime} 43.0^{\prime \prime}$ & $44^{\prime} 05.6^{\prime \prime}$ & $-24.8^{\prime \prime}$ & $3^{\prime} 58.7^{\prime \prime}$ \\
\hline
\end{tabular}

whereas for the other components the differences between the filtered and observed component value are computed.

\subsection{Example dataset Welfenschloss}

The data acquisition of the dataset Welfenschloss was performed with a phase-measuring laser scanner Zoller+Fröhlich Imager 5006, one Javad GNSS receiver Delta G3T with a Javad Grant G3T antenna, two Schaevitz LSOC-1 $1^{\circ}$ inclinometers and one Leica GRZ4 $360^{\circ}$ prism, which was tracked by a Leica TS30 robotic tacheometer. The two 3D positioning sensors were located on opposite sides on top of the laser scanner (see Figure 7). For a comparison of the two tracking approaches, an unique coordinate system for all measurements is required. This was established by setting up a geodetic network in the area in front of the main building of the LUH. The sculpture Lower Saxony Steed in front of the main building of the LUH was scanned in full from three different static stations. In the following only the comparison of the results of the two used 3D positioning sensors of one selected station will be shown. For a more comprehensive analysis of the measurement results see Paffenholz et al. (2010).

For the assessment of the attainable accuracy the positions, derived from the GNSS and the tacheom-

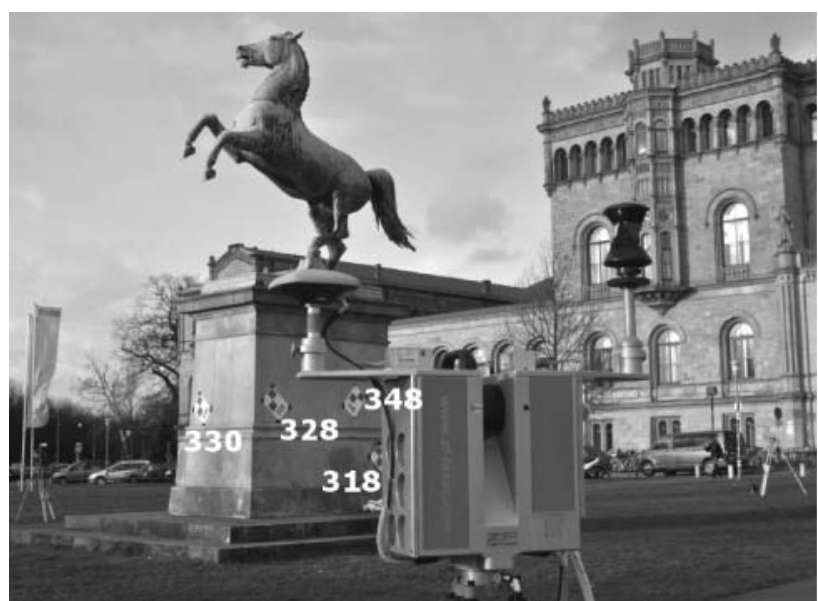

Figure 7: MSS with GNSS antenna (left) and prism (right) on top of the laser scanner in front of the sculpture of the Lower Saxony Steed (Lower Saxony's landmark) next to the main building of the LUH.

eter measurements, of four artificial control points on the sculpture (see Figure 7) were compared. Table 2 summarises the numerical values of the coordinate differences and spatial distances of the four selected control points. The distance between the station and the four targets is approximately $16 \mathrm{~m}$.

Table 2: Differences of control point coordinates on the sculpture caused by two different tracking approaches (GNSS measurements and prism tracked by robotic tacheometer) for the TLS measurements.

\begin{tabular}{lccrc}
\hline point no & $\mathrm{dx}[\mathrm{m}]$ & $\mathrm{dy}[\mathrm{m}]$ & $\mathrm{dz}[\mathrm{m}]$ & $\mathrm{ds}[\mathrm{m}]$ \\
\hline 328 & -0.002 & -0.034 & -0.038 & 0.051 \\
348 & -0.001 & -0.041 & -0.038 & 0.056 \\
318 & -0.001 & -0.042 & -0.038 & 0.057 \\
330 & -0.003 & -0.033 & 0.042 & 0.053 \\
\hline
\end{tabular}


As one can see, the tracking of both sensors come nearly to the same result. The differences between the coordinates are less than one decimetre. Furthermore, it can be noticed that the differences in the spatial distance are almost the same for this selected station and are a result of the uncertainty of the estimated azimuth per station, which is used for all targets.

Nevertheless, the above mentioned results show the operability of the geo-referencing strategy independent of the operating geodetic sensor. A comparison of the global reference control points and the coordinates resulting from the direct geo-referencing shows significantly larger differences. An increase of almost 1.5 times is noted, which is a central topic of the ongoing investigations.

\section{Conclusions and outlook}

This paper describes an integrated method for the direct geo-referencing of static terrestrial laser scans by tracking the circular motion of the laser scanner about its vertical axis with a $3 \mathrm{D}$ positioning sensor. This approach makes it possible to determine the laser scanner orientation without the use of an additional backsight target as used by Reshetyuk (2009). Furthermore, a new algorithm for the estimation of the transformation parameters is outlined. The transformation parameters are required for the direct geo-referencing of terrestrial laser scans. It is based on an AEKF which was modified and optimised from a previous approach used by the authors. In addition the horizontal motor steps of the laser scanner were used as observation data to improve the filter.

The topics for ongoing research include a tailored data aggregation step to smooth the high frequency trajectory positions to the TLS data rate and an improvement of the prediction method for position data with lower acquisition rates. In order to get appropriate a priori values for the variance factors and to gain a better understanding of the process noise, a variance component estimation in the Kalman filter will be implemented according to Wang et al. (2009). The first step is the estimation of a posteriori variance factor for the observations and the process noise. The second step will be a variance component estimation for the different observation types. In this special application further investigations are necessary of the dynamic behaviour of the inclinometers used as sensors in the MSS. This is particularly important since low cost inclinometers are integrated in the laser scanners.

\section{Acknowledgments}

Our warmest thanks go to Prof. C. Rizos and Dr. J. Rüeger for their useful suggestions.

\section{References}

Alkhatib, H., Paffenholz, J.-A. and Kutterer, H., Sequential Monte Carlo Filtering for nonlinear GNSS trajectories, in: Proceedings of the 7th Hotine-Marussi-Symposium, Rome, Springer, 2010.

Aussems, T., Position estimation of land vehicles by means of Kalman filtering from satellites and combined navigation observations (in German), PhD thesis, Publications of the Geodetic Institute of the Rheinisch-Westfälischen Technischen Hochschule Aachen, Aachen, 1999.

Besl, P. J. and McKay, N. D., A Method for Registration of 3-D Shapes, IEEE Transactions on Pattern Analysis and Machine Intelligence 14:2 (1992), 239-256.

Eichhorn, A., Analysis of dynamic deformation processes with adaptive KALMAN-filtering, Journal of Applied Geodesy 1:1 (2007), $9-15$.

Nelson, A. T., Nonlinear Estimation and Modeling of Noisy TimeSeries by Dual Kalman Filtering Methods, PhD thesis, Oregon Graduate Institute, 2000.

Paffenholz, J.-A., Alkhatib, H., Brieden, P. and Kutterer, H., Optimized Direct Geo-Referencing Strategy for a TLS-based MultiSensor-System, in: Grün, A. and Kahmen, H., editors, Optical 3D-Measurement Techniques IX, volume I, 2009.

Paffenholz, J.-A., Horst, S., Alkhatib, H. and Kutterer, H., On the tracking of a Laser Scanner for Geo-Referencing Tasks by Means of Geodetic Sensors, in: Schulze Lammers, P. and Kuhlmann, H., editors, 2nd International Conference on Machine Control \& Guidance - Proceedings, 2010.

Paffenholz, J.-A. and Kutterer, H., Direct Georeferencing of Static Terrestrial Laser Scans, in: Proceedings of the FIG Working Week in Stockholm, 2008.

Reshetyuk, Y., Self-calibration and direct georeferencing in terrestrial laser scanning, PhD thesis, Royal Institute of Technology, Stockholm, 2009.

Simon, D., Optimal State Estimation, Wiley, Hoboken, New Jersey, 2006.

Teunissen, P. J. G., Giorgi, G. and Buist, P. J., Testing of a new single-frequency GNSS carrier phase attitude determination method: land, ship and aircraft experiments, GPS Solutions (to be published) (2010), URL doi:10.1007/s10291-010-0164-x.

Vennegeerts, H., Martin, J., Kutterer, H. and Becker, M., Validation of a kinematic laserscanning system, Journal of Applied Geodesy 2:2 (2008), 79-84.

Wang, J.-G., Gopaul, N. and Scherzinger, B., Simplified Algorithms of Variance Component Estimation for Static and Kinematic GPS Single Point Positioning, Journal of Global Positioning Systems 8:1 (2009), 43-52.

Wanninger, L., Baseline processing program Wa1, 2009, www. wasoft.de/wa1/index.html.

Wübbena, G., The GPS Adjustment Software Package - GEONAP - Concepts and Models, in: Proceedings of the Fifth International Symposium on Satellite Positioning, 1989.

Zoller+Fröhlich GmbH, Technical data sheet IMAGER 5006, 2007. 


\section{Author information}

Jens-André Paffenholz, Hamza Alkhatib and Hansjörg Kutterer

Geodetic Institute, Engineering Geodesy and Geodetic Data

Analysis Section, Gottfried Wilhelm Leibniz Universität

Hannover, Germany

E-mail: paffenholz@gih.uni-hannover.de,

alkhatib@gih.uni-hannover.de, kutterer@gih.uni-hannover.de 